\title{
Event-related potentials from the user's perspective
}

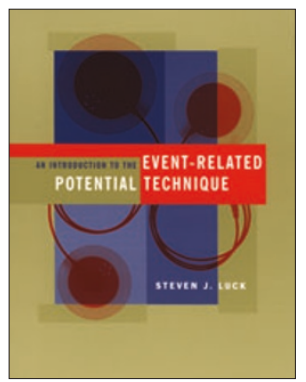

\section{An Introduction to the Event- Related Potential Technique}

\author{
By Steven J Luck
}

MIT Press, 2005

$376 \mathrm{pp}$, paperback, $\$ 40$

ISBN 0262621967

\section{Reviewed by Peter Hagoort}

In 1929, Hans Berger succeeded in measuring the electrical activity of the brain with the help of electrodes placed on the scalp. This technique has since been known as electroencepholography (EEG). In the sixties, different researchers started to record EEG in relation to cognitive tasks performed by the brain. By averaging the EEG signal time-locked to the relevant stimulus events, it became possible to identify a series of socalled event-related brain potentials (ERPs) that provide information about cognitive processes in the brain with a temporal resolution on the order of milliseconds. This method is known as the ERP technique.

Despite the more recent development of a number of new neuroimaging methods such as magnetoencephalograpy (MEG) and functional magnetic resonance imaging (fMRI), the ERP technique is still widely used. In part this is because recording ERPs is a lot cheaper than MEG and $\mathrm{FMRI}$, as a decent ERP setup requires an investment of less than a hundred thousand dollars. No wonder that these days one can find ERP setups in many departments of cognitive psychology, developmental psychology and even linguistics.

This success of the ERP method carries a certain risk, however. The early users of the ERP method had a solid background in psychophysiology with sufficient knowledge about the electrophysiological signals that were measured and the complexities of EEG recording and signal processing. Many of the new users lack this background. For them, a textbook with a solid introduction of the ERP method would be very helpful. Although some edited textbooks are around, so far an introduction written from an integrated user's perspective has been lacking. Luck has now filled this gap with An Introduction to the Event-Related Potential Technique.

Luck did a marvelous job in writing this book, largely due to the perspective of the user that he has taken as the guiding principle in composing his introduction. What do you need to know? Which problems do you face? What practical solutions are available if you have to

Peter Hagoort is at the F.C. Donders Centre for Cognitive Neuroimaging, Radboud University Nijmegen, P.O. Box 9101, Nijmegen 6500 HB, The Netherlands.

e-mail: peter.hagoort@fcdonders.ru.nl build up an ERP lab from scratch and want to use the ERP method for studying cognition? All these questions are answered in this book, in which Luck shares with the reader both his theoretical knowledge and his long practical experience in running an ERP facility. The author first describes how to design an ERP experiment and how to interpret the ERP waveforms. He provides examples of studies in different domains of cognition (such as visual attention and language) and summarizes the most relevant ERP components that are associated with these domains. From there, Luck takes the reader by the hand as he systematically discusses all the relevant aspects of the ERP method that are crucial for answering a particular research question. These issues include filtering, avoiding and rejecting or correcting artifacts, statistical analysis and graphical representations of the data, localization of ERPs, and the most important practicalities when setting up an ERP lab. Some chapters summarize the take-home message as a series of rules or strategies.

After discussing the relevant issues, he often provides recommendations. For instance in the chapter on localization, Luck warns the reader, "My basic conclusion is that ERP localization is extremely difficult, and it should be attempted only by experts and only when the solution space can be reduced by well-justified constraints, such as structural MRI data and the combination of electric and magnetic data." Luck stresses, rightly in my opinion, that the major contribution of the ERP method is related to the high temporal resolution it offers, which enables us to study the neural dynamics of cognition. In that context, he is skeptical about the current trend toward recording from increasing number of electrodes, beyond the 32 that are optimal to estimate the temporal profile of the relevant cognitive processes.

Luck has avoided the mathematics of electricity and magnetism as much as possible, but has added a useful appendix with the basic principles of electricity. He carefully avoids overloading the reader with advanced techniques, and focuses on the basics. At the same time, he succeeds in conveying to the reader that nothing comes for free. Using the ERP method to address research questions in cognitive neuroscience requires a minimum amount of expertise about the signals that one records, the possibilities and pitfalls encountered when analyzing these signals, and about what one can and —equally important—what one cannot conclude from the acquired data.

This excellent introduction to the ERP method should be read by all students entering the field of ERP research and by all researchers who want to extend their current methods with the recording of ERPs. Even for experienced ERP researchers, this text provides thoughtful comments and suggestions that one should carefully consider in optimizing one's research routines. An introduction tailored to the needs of a beginning user of the ERP method has been needed, and Luck has given us just the right one at the right time. In my lab, his text is already obligatory entrance reading for all students starting their ERP research. Very likely, in the near future, this introduction will serve the same purpose in many ERP labs around the world. 